\title{
ACE Inhibitory Effect of Strawberry
}

\section{Tahreem Javaid, Shahid Mahmood*, Wajiha Saeed, Muhammad Qamrosh Alam}

Institute of Food Science and Nutrition, University of Sargodha, Pakistan

*Corresponding Author: Shahid Mahmood, Institute of Food Science and Nutrition, University of Sargodha, Sargodha, Pakistan.

Received: June 20, 2019; Published: July 30, 2019

DOI: $10.31080 /$ ASNH.2019.03.0386

\begin{abstract}
Cardiovascular disease is the cluster of syndromes of blood vessels and heart that consist of hypertension, coronary heart disease, rheumatic heart disease, congenital heart disease, Cardiomyopathies, cerebrovascular disease, peripheral vascular disease and heart failure. Strawberry (Fragaria x ananassa) is produced in marketable scale because of the use of chemicals to retain the quality of fruit during storage and transport is prohibited, high perishability and short postharvest life. In, adults, the leading cause of cardiovascular disease is hypertension. It affects approximately $18 \%$ in Pakistan. As increase in blood pressure increases the threat for cardiovascular disease mortality. ACE inhibition is an important target for hypertension management. It was observed that the purified ellagitannins had higher ACE inhibitory activity than ellagic acid and strawberries (Lee, 2006).
\end{abstract}

Keywords: Cardiovascular Disease; Strawberry; Fragaria x ananassa

\section{Health}

Health is not just the absence of disease/infirmity, it is also the state of complete mental, physical and social well-being [1].

Cardiovascular disease

Cardiovascular disease is the cluster of syndromes of blood vessels and heart that consist of hypertension, coronary heart disease, rheumatic heart disease, congenital heart disease, Cardiomyopathies, cerebrovascular disease, peripheral vascular disease and heart failure [2].

\section{Hypertension}

A condition in which the blood vessels have stubbornly raised pressure. Blood is carried from the heart to all parts of the body in the vessels. It pumps blood into the vessels each time when the heart beats. It is a high or raised blood pressure. By pushing the force of blood against the walls of arteries as it is pumped by the heart, blood pressure is created. The harder the heart has to pump, the higher the pressure [3].

\section{Strawberry (Fragaria $x$ ananassa)}

Fragaria chiloensis and Fragaria virginiana are two native species which are hybrid of the major cultured strawberry (Fragaria $x$ ananassa). The breeders of strawberry (Fragaria $x$ ananassa) are engrossed on cultivating disease resistance, local adaptations, fruit quality and productivity. Botrytis cinerea, Colletotrichum spp., Phytophthora cactorum, Phytophthora Fragariae and Verticillium alboatrum are the major pathogens [4]. Strawberry (Fragaria $x$ ananassa) is produced in marketable scale because of the use of chemicals to retain the quality of fruit during storage and transport is prohibited, high perishability and short postharvest life. Due to high content of essential nutrients and beneficial phytochemicals, which seem to have relevant biological activity in human health, Strawberry (Fragaria x ananassa) is called an important fruit in Mediterranean diet.

Phytochemicals, anthocyanin and ellagitannins are the major antioxidant compounds. An individual phytochemical constituents of strawberries have been studied for their biological activities, hu- 
man intervention studies using whole fruits are still lacking [5]. In Italy, the history recording of Strawberry (Fragaria x ananassa) has been growing wild for over 2200 years as long ago as 234BC. Strawberry (Fragaria $x$ ananassa) were first cultivated was not introduced until late 13 century. In early1643, Native American growing strawberries were found by early settlers.

\section{Bioactive compound}

The strawberry (Fragaria $x$ ananassa) is a relevant source of bioactive compounds because of its high levels of vitamin $C$, phenolic constituents and folate [6]. Most of which express relevant antioxidant capacities. Strawberry (Fragaria $x$ ananassa) is a foundation of healthy, essential fatty acids because its seed oil is rich in unsaturated fatty acids (w72\% polyunsaturated fatty acids). Polyphenolic phytochemicals (flavonoids, phenolic acids, lignans, and tannins) is used as a variety of non-nutritive components. The bioactive compounds are specific focus on phytochemical compounds, primarily anthocyanins and ellagitannins. Phytochemicals are mainly represented by the extensive class of phenolic compounds that have many nonessential functions in huge biological potentialities in humans and in plants. Flavonoids (mainly anthocyanins, with flavonols and flavanols providing a minor contribution) is the major class of phenolic compound, which is followed by phenolic acids (hydroxybenzoic acids and hydroxycinnamic acids) and hydrolyzable tannins (ellagitannins and gallotannins) with the minor constituents as condensed tannins (proanthocyanidins) [7].

\section{Mineral profile}

\begin{tabular}{|l|c|c|}
\hline Type & Nutrient & Per 100g \\
\hline Proximate & Water(g) & 90.95 \\
\hline & Energy(kcal) & 32 \\
\hline & Protein(g) & 0.67 \\
\hline & Dietary fiber (g) & 2.0 \\
\hline & Ash (g) & 0.40 \\
\hline & Total lipid (g) & 0.30 \\
\hline & Carbohydrates(g) & 7.68 \\
\hline & Sucrose (g) & 0.47 \\
\hline & Sugars (g) & 4.89 \\
\hline & Glucose(g) & 1.99 \\
\hline Minerals & Fructose (g) & 2.44 \\
\hline & Calcium (mg) & 16 \\
\hline & Iron (mg) & 0.41 \\
\hline
\end{tabular}

\begin{tabular}{|c|c|c|}
\hline & Magnesium (mg) & 13 \\
\hline & Phosphorus (mg) & 24 \\
\hline & Potassium (mg) & 153 \\
\hline & Sodium (mg) & 1 \\
\hline & Zinc (mg) & 0.14 \\
\hline & Copper (mg) & 0.048 \\
\hline & Manganese (mg) & 0.386 \\
\hline & Selenium (mg) & 0.4 \\
\hline \multirow[t]{17}{*}{ Vitamins } & Vitamin $\mathrm{C}(\mathrm{mg})$ & 58.8 \\
\hline & Thiamin (mg) & 0.024 \\
\hline & Riboflavin (mg) & 0.022 \\
\hline & Niacin (mg) & 0.386 \\
\hline & $\begin{array}{c}\text { Pantothenic acid } \\
\text { (mg) }\end{array}$ & 0.125 \\
\hline & Vitamin B6 (mg) & 0.047 \\
\hline & Folate (mg) & 24 \\
\hline & Choline (mg) & 5.7 \\
\hline & Betaine (mg) & 0.2 \\
\hline & Vitamin B12 (mg) & 0 \\
\hline & $\begin{array}{c}\text { Vitamin A, RAE } \\
(\mathrm{mg})\end{array}$ & 1 \\
\hline & $\begin{array}{l}\text { Lutein p zeaxan- } \\
\text { thin (mg) }\end{array}$ & 26 \\
\hline & $\begin{array}{l}\text { Vitamin E, a- } \\
\text { tocopherol (mg) }\end{array}$ & 0.29 \\
\hline & b-tocopherol (mg) & 0.01 \\
\hline & g-tocopherol (mg) & 0.08 \\
\hline & d-tocopherol (mg) & 0.01 \\
\hline & $\begin{array}{l}\text { Vitamink phyllo- } \\
\text { quinone (mg) }\end{array}$ & 2.2 \\
\hline
\end{tabular}

Table 1

(US Department of Agriculture and Agriculture Research Service, 2010)

\section{Effect of strawberry (Fragaria $x$ ananassa) on Hypertension}

In, adults, the leading cause of cardiovascular disease is hypertension. It affects approximately $18 \%$ in Pakistan. As increase in blood pressure increases the threat for cardiovascular disease mortality. The clinical recommendations show the 2 stages of hypertension in person. Systolic blood pressure $\geq 160 \mathrm{mmHg}$ and diastolic blood pressure $\geq 100 \mathrm{mmHg}$ comprises a more extensive treatment. 
The 1 stages of hypertension is SBP $140-159 \mathrm{mmHg} / \mathrm{DBP} 90-99$ $\mathrm{mmHg}$. While, the ethnic disparities have been well documented in the prevalence of hypertension. The awareness, treatment and control within blood pressure stages.

To examine ethnic disparities in awareness, treatment and control of high blood pressure by hypertension stages. Although the treatment with stage 2 hypertension did not differ by ethnicity among persons. The stage 2 hypertension were treated with medication is less than $60 \%$. More efforts are needed to reduce barriers to accessing low-cost medication and health care, along with increasing adherence to clinical guidelines and clinicians' hypertension treatment knowledge. The marginal hypertension patients by recurrently have a hyperkinetic circulation that can be normalized with autonomic blockade of the heart and is neurogenic. The origin of central nervous system is aberration and due to decreased vagal inhibition of the heart and increased sympathetic stimulation. A patients categorized by norepinephrine and high plasma renin values clearly has a neurogenic hypertension. The blood pressure becomes normal after vascular alpha-adrenergic blockade and cardiac blockade. Since, many patients with marginal hypertension have a high cardiac output.

Most of the patients with more innovative hypertension have increased vascular resistance. The main source of ellagic acid derivatives in the diet is represented by Strawberry (Fragaria $x$ ananassa) more than $50 \%$ of all phenolic compounds found in the fruit [8]. Because of possible chemopreventive benefits, there is a particular interest in the firmness of the ellagic acid content in fruits. The satisfying approach available for type 2 diabetic patients is by delaying the absorption of glucose through the reduction of starch hydrolysis through strongly inhibiting intestinal glucose absorption by $\alpha$-glucosidase enzymes and slightly inhibiting pancreatic $\alpha$-amylase. Natural $\alpha$-glucosidase and $\alpha$-amylase inhibitors from fruits and vegetables could offer a good strategy to control the postprandial hyperglycemia [9]. It provide benefits without the side effects present in most available drugs such as flatulence, possibly diarrhea and abdominal distention [10].

It has been reported that individuals affected by diabetes have associated risk factors, including obesity, hypertension and dyslipidemia [11]. An important enzyme involved in maintaining vascular tension by two different reactions is Angiotensin I-converting enzyme Angiotensin I-converting enzyme (ACE). It catalyzes the conversion of the inactive angiotensin I into a powerful vasoconstrictor and inactivation of the vasodilator bradykinin, salt-retainer and angiotensin II which is conducive to lowering blood pressure [12]. The useful therapy in the control of blood pressure in hypertensive patients is considered as inhibition of enzyme, which is a macro vascular complication of type 2 diabetes. So, to evaluate the potential health benefits of the purified ellagitannins was the objective of this study from strawberries in relation to the in vitro inhibitory activity and antiproliferative activity of enzymes related to hypertension and type 2 diabetes like $\alpha$-glucosidase, ACE and $\alpha$-amylase. Many fruits, including strawberry (Fragaria $x$ anan$a s s a$ ) is known to contain high levels of soluble tannins, and has $\alpha$-amylase inhibitory properties. Neither ellagic acid nor gallic acid inhibited $\alpha$-amylase, indicating that other phenolic compounds are related to this functionality. The high blood pressure/ hypertension is one of the long-term complications of type 2 diabetes [13].

Many studies have reported the beneficial effects in hypertension of polyphenol-rich extracts not only by normalizing the blood pressure but also by retarding the development of hypertension [14]. ACE inhibition is an important target for hypertension management. It was observed that the purified ellagitannins had higher ACE inhibitory activity than ellagic acid and strawberries [15]. Besides, these results suggested that probably there is no synergistic effect of these compounds when present in food. There is evidence that some peptides naturally present in foods that could be responsible for such inhibitory activity or some water-soluble compounds other than the phenolics [16].

\section{Bibliography}

1. Conference IH. "Constitution of the World Health Organization. 1946". Bulletin of the World Health Organization 80.12 (2002): 983.

2. Pradeau C., et al. "Air Pollution and Activation of Mobile Medical Team for Out-Of-Hospital Cardiac Arrest". The American Journal of Emergency Medicine 33 (2015): 367-372.

3. Mehboudi MB., et al. "Inverse Association between Cigarette and Water Pipe Smoking and Hypertension in an Elderly Population in Iran: Bushehr Elderly Health Programme". Journal of Human Hypertension 31 (2017): 821. 
4. Hancock J F., et al. "Strawberries". In Temperate Fruit Crop Breeding. Springer, Dordrecht (2008): 393-437.

5. Giampieri F., et al. "The Strawberry: Composition, Nutritional Quality, and Impact on Human Health". Nutrition 28 (2012): 9-19.

6. Proteggente AR., et al. "The Antioxidant Activity of Regularly Consumed Fruit and Vegetables Reflects their Phenolic and Vitamin C Composition". Free Radical Research 36 (2002): 217-233.

7. Kähkönen M. P., et al. "Berry Phenolics and Their Antioxidant Activity". Journal of Agricultural and Food Chemistry 49 (2001): 4076-4082.

8. da Silva Pinto M., et al. "Functionality of Bioactive Compounds in Brazilian Strawberry (Fragaria× Ananassa Duch.) Cultivars: Evaluation of Hyperglycemia and Hypertension Potential Using In Vitro Models". Journal of Agricultural and Food Chemistry 56 (2008): 4386-4392.

9. Devi SA. "Smart Dietary Interventions and Prevention of Cognitive Decline with Aging". In Brain Aging and Therapeutic Interventions Springer, Dordrecht (2012): 253-266.

10. Bhandari MR., et al. " $\alpha$-Glucosidase and $\alpha$-Amylase Inhibitory Activities of Nepalese Medicinal Herb Pakhanbhed (Bergenia Ciliata, Haw.)". Food Chemistry 106 (2008): 247-252.

11. Narayan KV., et al. "Lifetime Risk for Diabetes Mellitus in the United States”. JAMA 290 (2003): 1884-1890.

12. Kwon YII., et al. "Evaluation of Clonal Herbs of Lamiaceae Species for Management of Diabetes and Hypertension". Asia Pacific Journal of Clinical Nutrition 15 (2006): 107-118.

13. Sunkara N and CH Ahsan. "Hypertension in Diabetes and the Risk of Cardiovascular Disease". Cardiovascular Endocrinology and Metabolism 6 (2017): 33-38.

14. Taubert D., et al. "Chocolate and Blood Pressure in Elderly Individuals with Isolated Systolic Hypertension". JAMA 290 (2003): 1029-1030.
15. Lee JE., et al. "Tyr-Pro-Lys, an Angiotensin I-Converting Enzyme Inhibitory Peptide Derived From Broccoli (Brassica Oleracea Italica)". Food Chemistry 99 (2006): 143-148.

16. Cheplick S., et al. "Clonal Variation in Raspberry Fruit Phenolics and Relevance for Diabetes and Hypertension Management". Journal of Food Biochemistry 31 (2007): 656-679.

\section{Volume 3 Issue 8 August 2019}

(C) All rights are reserved by Shahid Mahmood., et al. 